\title{
Intraspinal arachnoiditis and hydrocephalus after lumbar myelography using methylglucamine iocarmate
}

\author{
T. ST A E H E L I N J E N E N A N D O. H E I N \\ From the Department of Neurology, Vejle County Hospital, and the Department of Neurosurgery, \\ Odense University Hospital, Denmark
}

SUMMARY A 35 year old woman developed a severe meningeal reaction after lumbar myelography using the water-soluble contrast medium methylglucamine iocarmate. Three months after myelography the findings were a transverse spinal cord syndrome corresponding to the middle thoracic segments resulting from well developed leptomeningeal adhesions. This was combined with a noncommunicating hydrocephalus, probably the result of leptomeningeal fibrosis in the posterior fossa. After treatment with a ventriculoatrial shunt the patient is almost free of symptoms. A possible pathogenetic relationship between the contrast medium, the chronic leptomeningeal changes, and the symptoms of our patient is discussed on the basis of the literature.

Although myelography is one of the most valuable diagnostic tests in various spinal cord lesions, several of the contrast media used in this diagnostic procedure give rise to a broad spectrum of side effects. Among these the most common are acute or chronic leptomeningeal reactions (Autio et al., 1972; Ahlgren, 1973; Halaburt and Lester, 1973; Irstam and Rosencrantz, 1973).

Oil-based contrast media-for example, iophendylate, Pantopaque-which are mainly used in the cervicodorsal region, in cases of suspected spinal cord or nerve root compression, have an irritating effect on the medulla and the nerve roots. Their use is frequently accompanied by acute mild leptomeningeal inflammation, with an increase in cell and protein content of the cerebrospinal fluid. Although serious cases of chronic arachnoiditis or adhesive arachnoidal changes after the use of iophendylate are uncommon, they have been observed both in the upper intraspinal region and intracranially, and are occasionally followed by hydrocephalus with a lethal course (Tarlov, 1945; Mason and Raaf, 1962; Mayher et al., 1971; Christy et al., 1974). In all these cases the leptomeningeal changes were thought to be a result of a direct inflammatory effect of the iodised compound.

Address for correspondence and reprint requests: Dr T. Staehelin Jensen, Department of Neurosurgery, Århus University Hospital, 8000 Århus C, Denmark.

Accepted 5 August 1977
The water-soluble contrast media-for example, $\stackrel{\mathbb{P}}{\stackrel{\oplus}{\mathbb{2}}}$ 을 methylglucamine iothalamate (Conray) and $\mathrm{C}-$ methylglucamine iocarmate (meglumine iocar 웅 T) mate, Dimer-X)-are used mainly for investigation of the lumbar part of the spinal canal, giving morec detailed pictures than oil-based contrast media However, like the oily contrast media, these agents are more or less neurotoxic, and various complications may be observed after myelography with water-soluble contrast agents. In recent years delayed adhesive arachnoidal changes after the use of these substances have been reported more frequently (Autio et al., 1972; Ahlgren, 1973, 1975; Halaburt and Lester, 1973; Irstam and Rosencrantz, 1973). All arachnoidal changes observed to date have been located exclusively in the caudal region of the spinal canal (Ahlgren, 1973; Halaburt and Lester, 1973; Irstam and Rosencrantz, 1973; Slätis et al., 1974). This paper describes an unusual case of intracranial and midthoracic arachnoiditis in which the clinical symptoms appeared shortly after lumbar myelography with the water-soluble contrast medium meglumine iocarmate.

\section{Case report}

The patient was a 35 year old woman, with a history of good health, except for transient biliary tract symptoms in 1968.

In 1973 and 1974 she experienced transient re- 
lapsing lumbar pain, but was otherwise healthy and fit. In October 1974, without preceding trauma, she developed acute pains in the right lumbar region, radiating to the right leg. Three months later she was admitted to the local hospital with unaltered pain. Physical examination showed a slightly weakened right patellar reflex, but the neurological examination was otherwise normal. A lumbar disc herniation was suspected and lumbar myelography using meglumine iocarmate (Dimer-X) was carried out on 31 January 1975. Lumbar puncture and injection of the contrast medium was performed with the patient in a sitting position; the remaining part of the examination was made with the trunk elevated $15^{\circ}$. The cerebrospinal fluid was clear, colourless, contained no cells, and the protein content was 0.33 $\mathrm{g} / \mathrm{l}$. Dimer-X $5 \mathrm{ml}$ diluted with $4 \mathrm{ml}$ cerebrospinal fluid was injected. The contrast medium was not aspirated at the end of the examination.

During myelography, which showed normal conditions, the contrast medium was seen to move up to the level of the twelfth thoracic vertebra (Fig. 1).

After myelography the patient was kept in a sitting position. A few hours after the examination, while still resting with the trunk and head elevated, she complained of intense headache, nausea, and restlessness in her legs. At this time her temperature had fallen to $33^{\circ} \mathrm{C}$ and later rose steadily to $39.3^{\circ}$. The next day the patient was still in a poor condition; she was febrile with restless movements of all limbs, and a pronounced neck rigidity was noted. Apart from hypaesthaesia and hypalgesia in the perianal area, no neurological deficit was found. No fluid could be removed in repeated lumbar punctures. After eight days her temperature was normal, but there was still slight neck stiffness, and the patient complained of headache which persisted unchanged until she was discharged five weeks later.

During the subsequent months, the frequency and intensity of headaches increased. Three months after myelography she had bifrontal headache daily accompanied by repeated vomiting, and rapidly developing gait disturbances with limb weakness and an unsteady gait. When the patient was admitted to the neurological department of Vejle County Hospital in May 1975, physical examination revealed bilateral papilloedema (1-2 diopters). There was moderate spastic paresis of both legs and a bilateral sensory loss distal to the fourth thoracic dermatome. Her gait was spastic and ataxic, and a slight incoordination was also present in the upper extremities. A provisional clinical diagnosis was made of

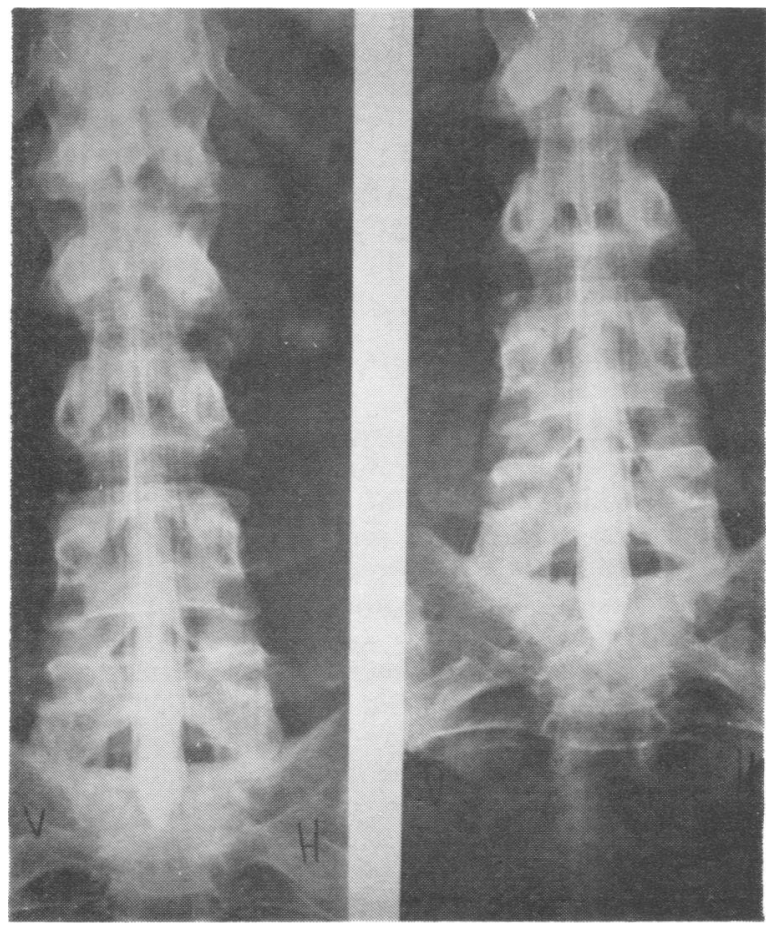

Fig. 1 Initial lumbar myelography with $5 \mathrm{ml}$ Dimer-X, showing well-filled root pockets and normal appearances of fila radicularia. 
a space-occupying lesion in the spinal canal and in the posterior fossa, and the patient was transferred to the neurosurgical department of Odense County Hospital, where suboccipital ethyl monoiodostearate (Duroliopaque) myelography revealed a complete obstruction at the level of the sixth thoracic vertebra (Fig. 2). Cerebrospinal fluid from the suboccipital level was clear and colourless; it contained $0.1 \mathrm{~g} / 1$ of protein and there were no abnormal cells.

\section{OPERATION}

Laminectomy of thoracic vertebrae 5-7 inclusive was performed on the day of admission. The arachnoid membrane was milky, greatly thickened and adherent, with pouch formation partly to the spinal cord and partly between the arachnoid and the dura mater. Adhesions continued both cranially and caudally. A biopsy sample of the

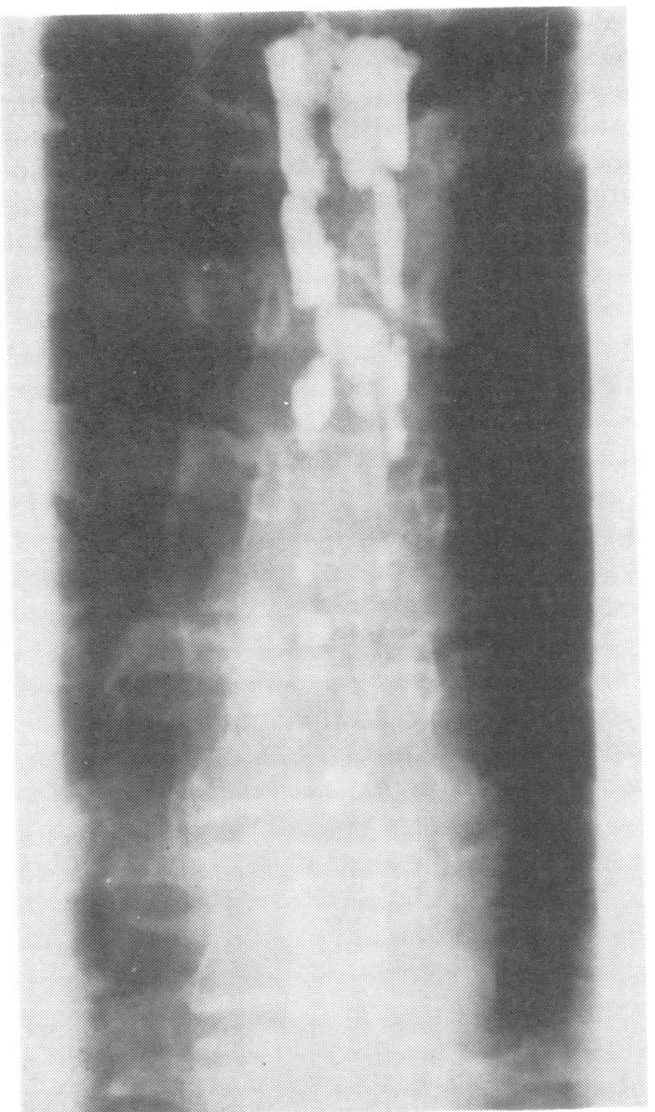

Fig 2 Positive contrast oil myelogram showing complete obstruction to flow at the level of the sixth thoracic vertebra. arachnoid membrane piece of tissue in which arachnoid and dura mater were fused was obtained. Focal cell proliferation could be seen at the junction of the two membranes. There were no malignant changes nor any sign of acute inflammation, and fungi and bacteria could not be demonstrated. The histological findings were thus typical of a chronic adhesive arachnoiditis.

During steroid treatment (dexamethasone), with a total dose of $200 \mathrm{mg}$ during and after the operation, there was a marked but transient improvement in the patient's condition so that she was able to walk, but three weeks after the operation there was a relapse. She had progressive paresis of the lower extremities and headaches, culminating in unconsciousness lasting seconds to minutes accompanied by explosive vomiting. Intraventricular pressure was monitored continuously and showed that these episodes of unconsciousness coincided with transient increases in pressure from $10-20 \mathrm{mmHg}$ to some $90 \mathrm{mmHg}$. There was progressive papilloedema (2-3 diopters), and fresh retinal haemorrhages were seen.

Ventriculography was carried out in June 1975 and showed that the supratentorial ventricular system was moderately dilated, whereas the fourth ventricle was filled and in normal position. No air passed into the cisterna magna or to the subarachnoid space as a whole, indicating a noncommunicating hydrocephalus.

A ventriculoatrial shunt (Hakim-CordisMedium) was implanted three days after ventriculography, and her condition gradually improved with intensive physiotherapy. Fifteen months later she is capable of walking with little difficulty, using a stick, has no headache, and is neurologically intact apart from a slight spastic paresis of the left lung.

\section{Discussion}

The patient presented with abrupt onset of neck rigidity, headache, drowsiness, and temperature disturbances, shortly after lumbar myelography with a water-soluble contrast agent which suggests that the performance of this procedure played an important pathogenetic role.

Several reports have confirmed the neurotoxic effect of these substances, and a series of side effects, usually transient and slight, such as headache, nausea, vomiting, dizziness, and fever have been reported (Gonsette, 1971; Lehtinen and Seppänen, 1972; Irstam, 1973; Ahlgren, 1975).

Our patient had clear indications of meningeal irritation after myelography. Irstam (1973) and Vik-Mo and Maurer (1975) reported a similar 


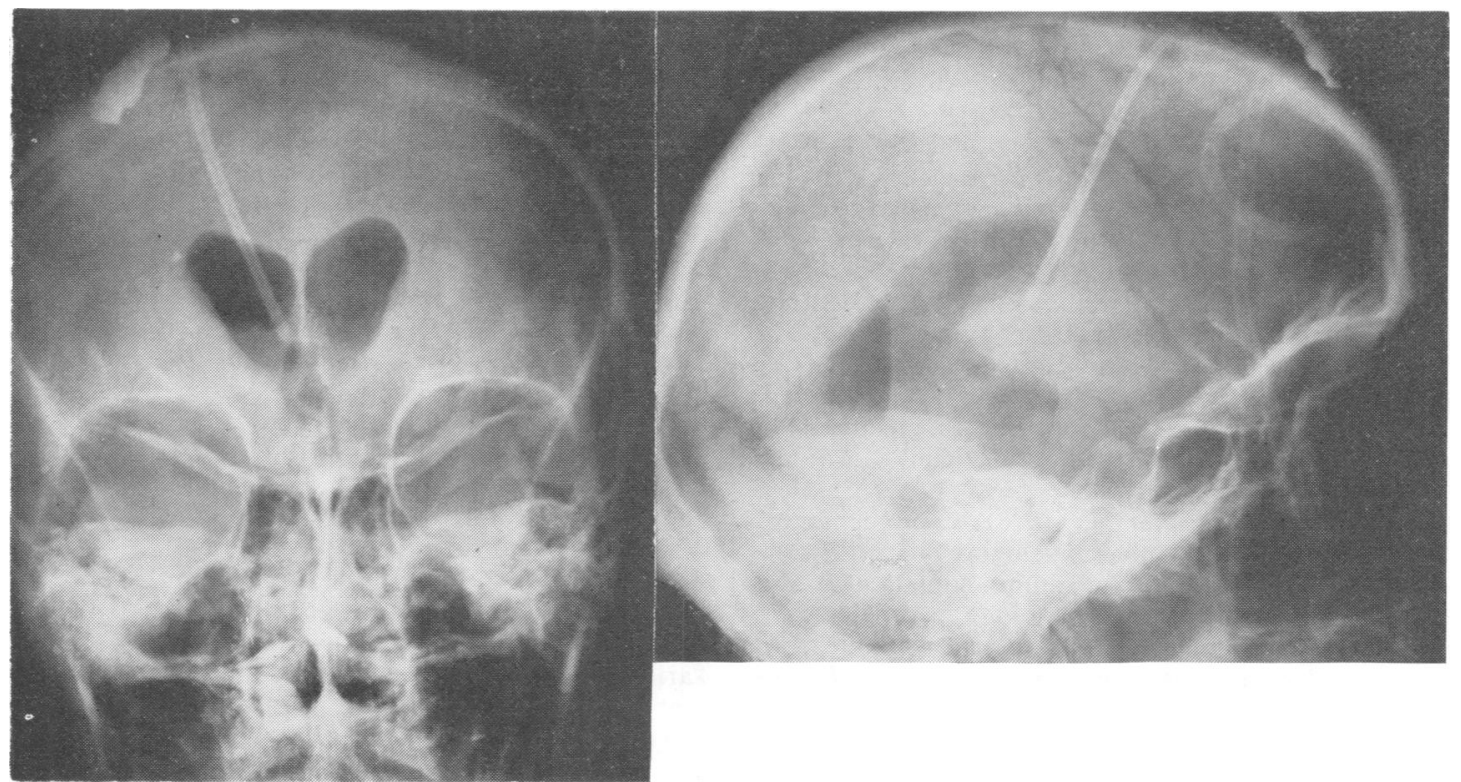

Fig. 3 Ventriculography, anteroposterior view (left), lateral view (right). The ventricular system is enlarged with no air passing to the basal cisterns or over the cerebral convexity.

complex of symptoms after lumbar myelography using Dimer-X. Sterile meningitis with moderate increases in cell and protein content of the cerebrospinal fluid was found in these cases. It is not possible to establish a diagnosis of aseptic meningitis in the present case, but the course of the disease makes it most likely.

In addition to acute leptomeningeal changes, water-soluble contrast media may also give rise to permanent leptomeningeal alterations characterised by considerable thickening of the leptomeninges and adhesions between dura and arachnoid mater (Ahlgren, 1973, 1975; Irstam and Rosencrantz, 1973; Rådberg and Wennberg, 1973; Slätis et al., 1974). Previously, the development of these leptomeningeal adhesions has only been demonstrated in the lower dural sac (Autio et al., 1972; Halaburt and Lester, 1973; Irstam and Rosencrantz, 1973; Suolanen, 1975), which is related to the fact that these media have been used exclusively in the lumbar region.

Three months after the primary lumbar myelography our patient manifested a transverse spinal cord syndrome, as a result of severe and extensive arachnoiditis in the midthoracic region of the spinal canal, together with an internal hydrocephalus, raised intracranial pressure, and papilloedema, due probably to an arachnoiditis in the posterior fossa with obstruction of the outlets of the fourth ventricle.
Although the exact mechanism for development of leptomeningeal adhesions is not known, we think it most probable that the arachnoiditis was caused by primary inflammatory reaction in the leptomeninges of the posterior fossa and in the spinal canal at the midthoracic level. Dimer-X was not seen during myelography higher than the level of the twelfth thoracic vertebra, but it is known from resorption studies (Brabrand et al., 1972; Irstam, 1973), that a subsequent diffusion of contrast medium to the circulating cerebrospinal fluid occurs during myelography, so that the medium also comes into contact with meninges in the cranial parts of the subarachnoid space.

We are grateful to Dr S. Melsen, Chief Neurologist, Vejle County Hospital, and to Dr J. E. Møller, Chief Pathologist, Gentofte, Denmark, for their advice.

\section{References}

Ahlgren, P. (1973). Long term side effects with water soluble contrast media: Conturex, Conray, Meglumin 282 and Dimer-X. Neuroradiology, 6, 206-211.

Ahlgren, P. (1975). Amipaque myelography. The side effects compared with Dimer-X. Neuroradiology, 9, 197-202. 
Autio, E., Suolanen, J., Norrbäck, S., and Slätis, P. (1972). Adhesive arachnoiditis after lumbar myelography with meglumine iothalamate (Conray). Acta Radiologica (Diagnosis), 12, 17-24.

Brabrand, H., Wessmann, H. D., and Wenker, $H$. (1972). Experimentelle untersuchungen über die elimination und neurotoxizität eines neuen, wasserlöslichen kontrastmittels zur lumbosakralen myelographie. Der Radiologe, 12, 66-68.

Christy, G., Scialfa, G., Di Pierro, G., and Tassony, A. (1974). Visual loss: a rare complication following oil myelography. Neuroradiology, 7, 287-290.

Gonsette, R. (1971). An experimental and clinical assessment of water-soluble contrast medium in neuroradiology. A new medium-Dimer-X. Clinical Radiology, 22, 44-56.

Halaburt, H., and Lester, J. (1973). Leptomeningeal changes following lumbar myelography with watersoluble contrast media (meglumin iothalamate and methiodal sodium). Neuroradiology, 5, 70-76.

Irstam, L. (1973). Side effects of water-soluble contrast media in lumbar myelography. Acta Radiologica (Diagnosis), 14, 647-656.

Irstam, L., and Rosencrantz, M. (1973). Water soluble contrast media and adhesive arachnoiditis. I. Reinvestigation of non-operated cases. Acta Radiologica (Diagnosis), 14, 497-506.

Lehtinen, E., and Seppänen, S. (1972). Side effects of Conray Meglumin 282 and Dimer-X in lumbar myelography. Acta Radiologica (Diagnosis), 12, 12 16.

Mason, M. S., and Raaf, J. (1962). Complications of Pantopaque myelography: case report and review. Journal of Neurosurgery, 19, 302-311.

Mayher, W. E., Daniel, E. F., and Allen, B. M. (1971). Acute meningeal reaction following pantopaque myelography. Journal of Neurosurgery, 34, 396.

Rådberg, C., and Wennberg, E. (1973). Late sequelae following lumbar myelography with water-soluble contrast media. Acta Radiologica (Diagnosis), 14, 507-511.

Slätis, P., Autio, E., Suolanen, J., and Norrbäck, S. (1974). Hyperosmolality of the cerebrospinal fluid as a cause of adhesive arachnoiditis in lumbar myelography. Acta Radiologica (Diagnosis), 15, $619-629$.

Suolanen, J. (1975). Adhesive arachnoiditis following myelography with various contrast media. Neuroradiology, 9, 73-78.

Tarlov, I. M. (1945). Pantopaque meningitis disclosecl at operation. Journal of the American Medical Association, 129, 1014-1016.

Vik-Mo, H., and Maurer, H. J. (1975). Meningeal reactions following myelography. Effects of detergent washing agent. Acta Radiologica (Diagnosis), 16, 39-42. 